\title{
A TEORIA DOS COMPLEXOS CULTURAIS: UMA PERSPECTIVA JUNGUIANA DO SOCIAL
}

\section{Cauan Esplugues Silva}

Universidade Federal do Paraná

\section{Carlos Augusto Serbena}

Universidade Federal do Paraná
Recebido em: 07/04/2020

$1^{\text {a }}$ revisão em: 02/11/2020

$2^{a}$ revisão em: 16/02/2021

Aceito em: 12/05/2021

\section{RESUMO}

Este artigo realiza uma análise conceitual da teoria dos Complexos Culturais, nas obras de Samuel Kimbles e Thomas Singer. A teoria dos Complexos Culturais pode ser utilizada como uma forma de análise de conflitos intergrupais, é essencialmente a descrição de uma sociologia interna. Esta revisão tem como objetivo apresentar uma síntese atual e crítica do conceito. Temas como integração, telos, e a presença dos Complexos Culturais na prática clínica e algumas críticas ao conceito são apresentados. Percebe-se a presença de conceitos complementares que buscam dar suporte a esta teoria. Por fim, observa-se a manutenção de uma estrutura base deste conceito, ainda que contribuições tenham sido constatadas ao longo das obras analisadas, principalmente na utilização de conceitos que buscam complementar a descrição da dinâmica dos Complexos Culturais.

Palavras-chave: psicologia analítica; complexo cultural; inconsciente cultural; conflito intergrupal. 


\section{THE CULTURAL COMPLEX THEORY: A JUNGIAN SOCIAL PERSPECTIVE}

\section{ABSTRACT}

This paper conducts a conceptual analysis of the theory of Cultural Complexes in the works of Samuel Kimbles and Thomas Singer. A theory of Cultural Complexes can be used as a form of intergroup conflict analysis, describing a description of an internal sociology. This review aims to present a current critique and critique of the concept. Integration themes, telos, and the presence of cultural complexes in clinical practice and some critiques of the concept are presented. Note the presence of complementary concepts that seek to support this theory. Finally, it is observed the maintenance of a basic structure of this concept, although the contributions have been verified throughout the analyzed works, mainly in the use of concepts that seek to complement the description of the applications of the Cultural Complexes.

Keywords: analytical psychology; cultural complex; cultural unconscious; intergroup conflict. 


\section{LA TEORÍA DE LOS COMPLEJOS CULTURALES: UNA PERSPECTIVA JUNGUIANA DE LO SOCIAL}

\section{RESUMEN}

Este artículo realiza un análisis conceptual de la teoría de los Complejos Culturales, en las obras de Samuel Kimbles y Thomas Singer. La teoría de los Complejos Culturales puede ser utilizada como una manera de analizar los conflictos intergrupales, es esencialmente una descripción de una sociología interna. Esta revisión tiene por objetivo presentar una síntesis actual y crítica del concepto. Temas como integración, telos, y la presencia de los Complejos Culturales en la práctica clínica y algunas críticas al concepto son presentadas. Se percibe la presencia de conceptos complementarios que buscan dar soporte a esta teoría. Por fin, se observa la manutención de una estructura base de este concepto, aunque contribuciones tengan sido constatadas a lo largo de las obras analizadas, principalmente en la utilización de conceptos que buscan complementar la descripción de la dinámica de los Complejos Culturales.

Palabras clave: psicología analítica; complejo cultural; inconsciente cultural; conflicto intergrupal. 


\section{INTRODUÇÃO}

A psicologia analítica é conhecida principalmente por suas contribuições na prática clínica, por meio das noções teóricas construídas por Carl Jung, estando em constante discussão e desenvolvimento. De fato, uma das preocupações de Jung era construir uma teoria que possibilitasse movimento constante, diálogo com outras áreas do conhecimento, a exemplo de suas participações em Eranos ${ }^{1}$.

Um dos desdobramentos da psicologia analítica foi a preocupação com os movimentos sociais, mudanças políticas, o impacto das guerras mundiais na psique, como a mobilização psíquica de Hitler na Alemanha dos anos 30 do século XX. Algumas dessas análises estão presentes no volume 10 das obras de Jung, Civilização em Transição. Este é o volume que concentra por excelência as suas análises sociais e culturais.

Algumas de suas análises expressavam a preocupação com a massificação do homem, sendo o processo de individuação um caminho possível para a diferenciação da massa. A sua vivência nas guerras mundiais refletiu na sua teoria já que, para Jung, é justamente tornar-se um ser singular que possibilita a vivência consciente no coletivo. A análise destas mobilizações de massa, como a ascensão do nacional-socialismo na Alemanha, partia da teoria dos arquétipos, como no texto "Wotan", de 1936.

Apesar disso, Joseph Henderson (1990) percebeu que havia uma lacuna, ou um aspecto pouco explorado, no desenvolvimento da teoria junguiana: o nível cultural da psique. Assim, Henderson propôs o conceito de Inconsciente Cultural, como uma camada intermediária entre o Inconsciente Pessoal e o Inconsciente Coletivo. Contudo, o conceito de Inconsciente Cultural não propõe uma dinâmica para a análise cultural e social. Nesse sentido, Samuel Kimbles $(1998,2000)$ ampliou a utilização da teoria dos complexos ao nível grupal, entendendo que o Inconsciente Cultural é composto por Complexos Culturais, possibilitando assim essa análise.

A teoria dos Complexos Culturais é uma elaboração contemporânea no campo de estudos junguiano, sendo Samuel Kimbles e Thomas Singer os seus principais autores. Ela busca propor uma nova perspectiva nas análises culturais, sociais e grupais, a partir da psicologia analítica. Sua preocupação está na descrição da dinâmica dos conflitos intergrupais.

Alguns autores junguianos propuseram anteriormente estudos sociais, políticos e culturais, como Andrew Samuels (1989/1992, 1993/1995, 2001/2002), Michael Vannoy Adams (1996). Outros como Walter Odajnyk (1976/2007) e Ira Progoff (1953) apresentaram, respectivamente, as ideias de Jung sobre política e o seu significado para as ciências sociais.

No Brasil, há vários trabalhos que comentam sobre a análise social a partir da teoria junguiana. Alguns autores como Roberto Gambini $(2000,2003)$ e Dias e Gambini 
(1999), escreveram sobre o legado psicológico da formação do Brasil e da nossa identidade nacional. Araujo (2002) afirma sobre a necessidade de se pensar a psicologia analítica no Brasil por meio da ideia de Inconsciente Cultural e na necessária incursão desta na cultura afro-brasileira.

Alguns trabalhos versam diretamente sobre os Complexos Culturais brasileiros, como Denise Ramos (2004) e Camila Novaes (2016), ao falarem sobre corrupção. Walter Boechat $(2009,2012,2016,2018)$ escreve sobre racismo no Brasil e Ramos (2010, 2011, 2012) escreve sobre a elaboração do trauma da escravidão. Alguns trabalhos têm se dedicado a discussão sobre o que Gambini (2000) nomeou como "Alma brasileira", discorrendo também sobre as questões raciais e indígenas no Brasil (Boechat, 2014; Oliveira, 2018, 2020). O elevado número de publicações nas últimas duas décadas sobre Complexo Cultural demonstra a necessidade de se delinear este conceito, já que não foram encontrados trabalhos anteriores com tal proposta. Para isso, retomar as suas bases conceituais, as concepções de Jung sobre cultura e o coletivo, bem como apresentar os desdobramentos nas obras de Thomas Singer e Samuel Kimbles se fazem necessários. Este trabalho se propõe justamente a realizar uma análise conceitual crítica do conceito de Complexo Cultural baseado nas obras dos referidos autores, Kimbles e Singer.

\section{METODOLOGIA}

A análise conceitual se caracteriza pelo exame interno e teórico de "conceitos nucleares da teoria, seus significados e suas gramáticas" (Machado, Lourenço, \& Silva, 2000, p. 23). A partir dessa forma de pesquisa pode-se avaliar os conceitos por meio de seus aspectos históricos, epistemológicos, filosóficos, podendo assumir diferentes níveis de análise da teoria ou texto psicológicos. Sua finalidade é identificar a compreensibilidade de uma teoria, a funcionalidade dos seus termos e o seu significado dentro de seu corpo teórico, com o objetivo de favorecer a comunicação, reduzindo suas ambiguidades.

Os seguintes textos foram selecionados devido a sua relevância na discussão conceitual sobre a teoria dos Complexos Culturais, tanto em sua construção, como em crítica ao conceito. Foram analisadas as obras de Singer (2002, 2003, 2006a, 2006b, 2006c, 2007, 2009, 2010, 2012a, 2012b, 2012c, 2013a, 2013b, 2014a, 2014b, 2015, 2016a, 2016b, 2016c, 2016d, 2017, 2018a, 2018b), Kimbles (1998, 2000, 2003b, 2004, 2006, 2007, 2014, 2016 e 2017), Singer e Kimbles (2004a, 2004b) e Singer e Kaplinsky (2010), bem como os textos de Lu (2013a; 2013b), Graaff (2018) e Samuels (2010) sobre Complexo Cultural. Referente as publicações de Singer, não foi possível a análise do texto "A Personal Meditation on Politics and the American Soul", devido a impossibilidade de acesso ao artigo. Thomas Singer e Samuel Kimbles são os principais teóricos deste conceito, apresentando tanto análises a partir deste conceito como contribuições para a consolidação do mesmo. Foram agregados os textos de Kevin Lu e Johann Graaff por serem artigos que criticam o conceito de Complexo Cultural, contribuindo na discussão e nos contrapontos apresentados a esta teoria. 
Buscou-se construir, a partir desses textos, uma análise conceitual da teoria dos Complexos Culturais - e de seus conceitos predecessores, Complexo e Inconsciente Cultural. Esta revisão do conceito se deu pela leitura prévia e posterior categorização. Reuniram-se as informações nas seguintes categorias: definição conceitual; conceitos complementares que auxiliassem na descrição da dinâmica dos Complexos Culturais, tais como telos, narrativas fantasmas, defesas arquetípicas do espírito coletivo, entre outros; desdobramentos do conceito no campo junguiano, bem como a sua implicação na prática clínica; e, as críticas realizadas à teoria dos Complexos Culturais.

\section{TEORIA DOS COMPLEXOS}

Como conceito fundante na construção da Psicologia Analítica, a teoria dos Complexos proposta por Carl Gustav Jung tomou forma e importância na primeira década de 1900. Após terminar a sua formação em Medicina, na Basiléia, Jung passa a atuar como psiquiatra no Hospital Psiquiátrico de Burghölzli. O contexto psiquiátrico da época - com autores como Pierre Janet, Emil Kraepelin, Gustav Aschaffenburg, Théodore Flournoy, Alfred Binet - influenciaram Jung em suas primeiras pesquisas (Shamdasani, 2012).

Shamdasani (2012), na introdução do Liber Novus (Livro vermelho), sintetiza as informações sobre o momento de Jung em Burghölzli. Ao retornar de seu período em Paris, em que trabalhou com Pierre Janet, Jung trabalhou em colaboração com Franz Rinklin em uma pesquisa com análise de associações linguísticas.

O teste de associação de palavras já havia sido utilizado por importantes psiquiatras, como Wundt, Kraepelin e Aschaffenburg. Jung aliou a aplicação deste teste aos aspectos teóricos de Flournoy e Janet. Este projeto tinha por objetivo realizar um vasto estudo de associações de pessoas 'sadias' no Burghölzli (Jung, 1904/2012, §1). Ao longo do experimento, percebeu-se que algumas reações associativas estavam sob influência de outros elementos que não só a atenção, um dos itens avaliados. Assim, algumas dessas "falhas de reação", seja nos lapsos de linguagem ou na prolongação da resposta, apresentavam grande carga emocional. Estes núcleos afetivos que se constelavam foram entendidos como complexos de tonalidade afetiva.

O livro 'Estudos Psiquiátricos' apresenta algumas referências ao que veio a ser entendido como conceito de complexo. Em seu estudo sobre um caso de sonambulismo, Jung explica sobre as personalidades subconscientes de uma médium a partir da ideia de desagregação de complexos psíquicos (Jung, 1902/2013, §93) - a médium analisada era Helene Preiswerk, prima de Jung. Em outro estudo, de 1903, sobre a simulação de distúrbio mental, Jung (1903/2013) retoma as ideias de Pierre Janet sobre a emoção e seus efeitos a memória para analisar as suas pesquisas com os testes de associação de palavras. Comentando sobre o efeito dissociativo das emoções a consciência, ele enfatiza o seu aspecto unilateral e autônomo (\$318-319; §339). As emoções têm uma influência 
perturbadora sob a consciência, principalmente no curso de pensamento com carga emocional, fazendo com que outros cursos do pensamento se mantenham obscurecidos (Jung, 1904/2013, §423). No ano seguinte, ao escrever sobre criptomnésia, Jung comenta sobre pensamentos com forte carga emocional, que se mantém na psique de uma maneira inconsciente. Neste momento, formula a ideia de que o inconsciente seja composto por diferentes complexos e que a maior parte dos elementos psíquicos sejam inconscientes, sendo o consciente apenas uma de suas partes (Jung, 1905/2013, §169-172).

A partir disso, podemos entender a teoria dos complexos como um conceito basal na prática clínica junguiana. Definido por Jung (1934/2013) como uma imagem com elevado grau de autonomia e relativa a "uma determinada situação psíquica de forte carga emocional e, além disso, incompatível com as disposições ou atitude habitual da consciência" (\$201). Ele possui um núcleo arquetípico e uma coerência interna, constituindo uma totalidade que, quando constelada, tende a distorcer as percepções e compreensão da consciência egóica e interferir no fluxo desta.

Para Jung (1934/2013), os complexos, como componentes do inconsciente pessoal, possuem algumas características, como: autonomia, inconsciência e repetição. Eles possuem energia própria, quanto mais inconsciente (ou distante do complexo do ego) ele for, maior será a sua autonomia em relação a consciência. Ele se expressa também a partir de sintomas, como pensamentos obsessivos e na formação dos sonhos, que para ele são a via régia para o inconsciente.

A clínica junguiana dos complexos entende que a produção psíquica de sintomas, a formação dos sonhos ou o próprio fio que tece e enreda a psique nas relações, tem como base os complexos. Eles envolvem a consciência em sua própria narrativa e perspectiva de vivenciar e sentir as relações. A finalidade da consciência é estabelecer uma relação com estas unidades psíquicas ideo-afetivas, entendendo que elas não cessam e que elas nos acompanham na jornada volúvel do processo de individuação. Assim, este conceito possui importância fundamental na prática clínica da psicologia junguiana clássica. Restrito somente a análise do inconsciente pessoal, para Jung, os eventos coletivos partiam de uma perspectiva arquetípica, como será descrito adiante no seu texto sobre Wotan. A ampliação da teoria dos complexos para a dimensão grupal é uma proposição recente, pertencente a teoria dos Complexos Culturais. Mas, antes de adentrarmos no conceito principal, será analisado a concepção de Jung sobre o coletivo e a cultura, além do conceito de Inconsciente Cultural.

\section{CULTURA, COLETIVO E INCONSCIENTE CULTURAL}

A construção teórica da psicologia analítica tem por influência os estudos de Jung sobre diferentes culturas, como uma maneira de validar o seu conceito de Inconsciente Coletivo. A validação se dava na demonstração de mitos, das mais 
diversas origens, que compartilhavam determinadas características, dando base ao conceito de arquétipo. O livro 'Transformações e Símbolos da Libido', de 1912, foi justamente a primeira contribuição de Jung neste sentido. Ainda que não houvesse desenvolvido especificamente o conceito de arquétipo neste trabalho, aparecendo em sua obra somente em 1919 (Jung, 1919/2013, pp. 72-82).

Ao falar de cultura, parto de duas referências. A primeira, Clifford Geertz (1973/2008), antropólogo estadunidense, que entende que o homem está inserido em uma teia social de significados que ele mesmo tece, sendo a cultura então "um padrão historicamente transmitido de significados incorporados em símbolos, um sistema de concepções herdadas expressas em formas simbólicas por meio das quais os homens comunicam, perpetuam e desenvolvem seu conhecimento e atitudes em relação à vida" (p. 66). A antropologia interpretativa de Geertz busca entender os sentidos internos de uma sociedade. Mais do que a mera expressão dos comportamentos e descrição objetiva, o seu foco é na ação simbólica da cultura. A segunda referência é James Hillman, que se refere a cultura a partir de um olhar imaginal, associando-a a "imaginação, crenças, sanções e valores coletiva e inconscientemente compartilhados" (Hillman, 1986, p. 29, tradução nossa).

Ainda que a discussão sobre o conceito de cultura seja extensa e complexa na antropologia e sociologia, utilizarei este breve recorte como guia e definição do que nomeio por cultura. Aliando estas duas concepções, a cultura pode ser entendida como padrões coletivamente compartilhados de ideias, fantasias, comportamentos, formas de relação, que se expressam de maneira consciente e inconsciente.

Dito isto, voltamos a Jung. A abordagem de Jung, em relação as questões culturais, possui ao menos três vertentes:

Primeiro, uma sensibilidade à alienação de muitos ocidentais de suas raízes primitivas e instintivas. Segundo, em sua conceituação do inconsciente coletivo, ele fez uma série de suposições que tinham implícito dentro delas um privilégio de atitudes e valores ocidentais, mas também uma invalidação de culturas tradicionais. Por fim, o conceito de inconsciente coletivo foi definido de forma a não permitir espaço para que a matriz cultural tivesse seu próprio campo de ação coexistente com as camadas pessoais e arquetípicas (Singer \& Kimbles, 2004b, p. 181, tradução nossa).

Explorando mais a segunda questão, Waldron (2003) argumenta que através de sua estrutura teórica do inconsciente coletivo, Jung "liga a psique das culturas primitivas e das crianças ao inconsciente" e "ao processo evolutivo da humanidade, que ele achou comparável ao desenvolvimento evolutivo da consciência" (p.37). Sustentando a "visão de que o 'primitivo' é incapaz de reflexões pessoais, podendo fazer frente ou ir contra o coletivo". Assim, Waldron (2003) entende que "a noção de inconsciente coletivo, como conceituada por Jung, também nega, em certa 
medida, a crença de que a cultura pode ser de propriedade exclusiva de qualquer grupo de pessoas" (pp. 39-40, tradução nossa).

Com base nas afirmações de Waldron (2003), Jung demonstrava implicitamente uma homogeneidade, uma universalidade humana com o conceito de Inconsciente Coletivo, havendo menor interesse nas particularidades culturais e históricas. O que foi afirmado também por Shamdasani (2011), implícito no texto de Jung (1916/2015) "A estrutura do inconsciente", que a psique coletiva universal possuía maior determinação que a relação do indivíduo com as dimensões coletivas da família e da raça. Neste sentido, também há outro aspecto a ser considerado nos escritos de Jung, a sua utilização da ideia de coletivo.

A relação entre indivíduo e coletivo, para Jung, é relacionado em sua teoria a partir dos conceitos de individuação, psique coletiva e massificação, além das influências teóricas de Jung descritas por Sonu Shamdasani (2011). Em 1916, Jung (1916/2015) entendia que a psique coletiva era composta pelas partes inferiores da função mental. Para que houvesse o desenvolvimento individual, deveria haver a repressão da psique coletiva, que era entendida como uma ameaça ao indivíduo. A contaminação pela psique coletiva resultava em uma imitação, sugestionando atitudes e produzindo contágio mental - processo de massificação, em que o indivíduo passa a ser um sujeito indiferenciado da massa.

No texto 'Adaptação, individuação e coletividade', Jung (1916/2012) entende que individuação e coletividade são dois pares de opostos, e a partir da adaptação que o indivíduo poderá se relacionar e corresponder as exigências internas e externas. Ainda que responder as condições internas (do inconsciente) seja fato fundamental, sem o retorno do indivíduo a coletividade e uma conformação coletiva, não será possível ocorrer uma mediação entre estes dois polos. Um dos caminhos indicados por Jung (1916/2012) é por meio do amor a outra pessoa, um "amor verdadeiro" em que a outra pessoa se torna "uma representante muito forte do inconsciente" (§ 1105), ou seja, é Eros quem pode colocar em confluência o eu e o Outro.

Em 'Tipos Psicológicos', Jung (1920/2013) comenta a relação entre individuação e o coletivo, pressupondo que esta deva ocorrer "de maneira mais intensa e abrangente" (§ 853), evitando o isolamento. Em 'Um mito moderno sobre as coisas vistas no céu', Jung (1953/2013) reafirma que "contra o efeito massificante de todas as medidas coletivas, existe somente um meio: a acentuação e a elevação de valor do indivíduo" (§ 719).

Outro aspecto relativo ao coletivo, para Jung (1925/2014), é apresentado em sua "geologia" da personalidade, nos seminários de 1925, em que discorre de maneira breve sobre um diagrama de diferentes camadas da personalidade. Este diagrama é composto por influências do núcleo familiar, clãs, nações, grandes grupos, como pode ser observado na figura 1. Ao propor isso, Jung (1925/2014) entende que há uma influência destas diferentes camadas na constituição da psique. 


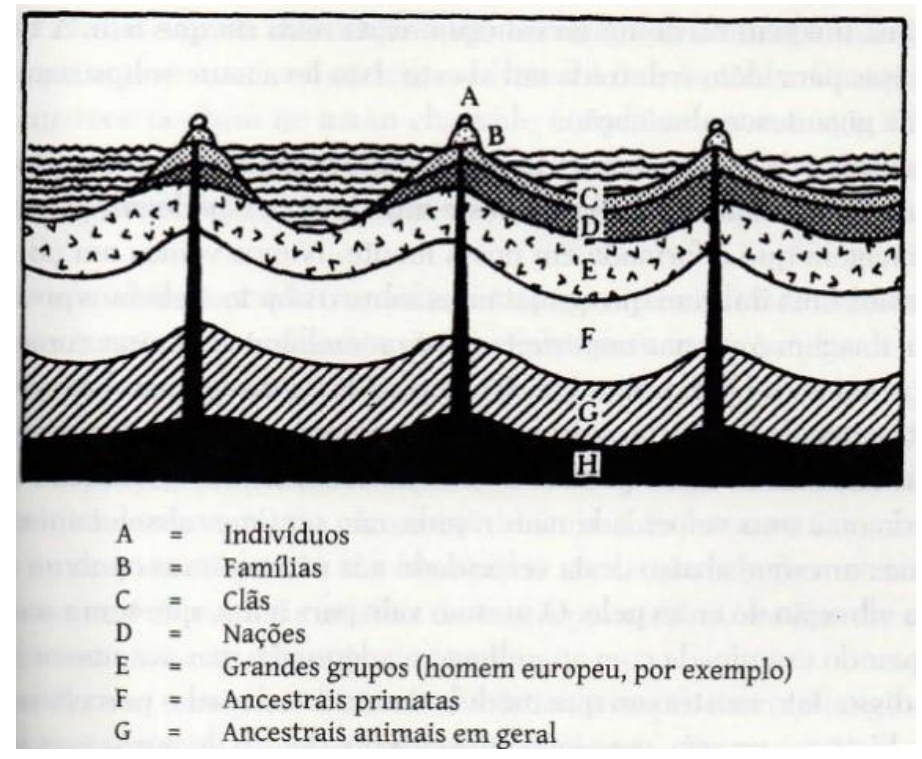

Figura 1- "Geologia" da personalidade para Jung e suas camadas

Estas são algumas das contribuições de Jung para se entender a relação entre indivíduo e coletividade, contudo o conceito de 'coletivo' não tem uma conotação positiva no léxico junguiano, justamente pela preocupação de Jung pela contaminação da psique coletiva, principalmente por sua vivência das guerras mundiais e o impacto psicológico que ela teve principalmente na Europa (Singer, 2003).

Uma análise do coletivo, realizada por Jung (1936/2012), pode ser vista em seu artigo "Wotan", ao analisar a ascensão do nacional-socialismo na Alemanha, tendo como figura principal Adolf Hitler e sua capacidade de mobilização da população. Wotan é um deus da mitologia nórdica da "tormenta e efervescência, desencadeador das paixões e das lutas e, além disso, mago poderoso e artista das ilusões, ligado a todos os segredos de natureza oculta" (pp. 15-16). Esta forma de análise considerava a desconexão daquela nação com os seus aspectos instintivos enquanto possessão da psique coletiva por um deus antigo daquela região. A análise de Jung se baseava, assim, nos aspectos arquetípicos da psique.

A palavra 'coletivo' cai, na maioria das vezes, na sombra do campo junguiano. Singer (2012c) comenta que este é um conceito confuso para alguns autores junguianos. Como forma de trazer uma definição sobre psique coletiva, o autor a entende como algo além da soma das psiques individuais, como se houvesse uma dinâmica autônoma e independente dos indivíduos, se expressando por eles.

Outro conceito explorado neste trabalho é de autoria de Joseph Henderson, médico e analista junguiano, que apresentou algumas produções sobre a relação entre indivíduo, psique e cultura. As suas contribuições passam pela ideia de arquétipo cultural, de atitudes culturais e de Inconsciente Cultural. A proposição de Samuel Kimbles dos Complexos Culturais tem origem conceitual na teoria dos 
complexos de Jung e de Inconsciente Cultural, de Henderson. Este autor define o conceito de Inconsciente Cultural como:

uma área da memória histórica entre o Inconsciente Coletivo e o padrão manifesto da cultura. Pode ser incluso ambas as modalidades, consciente e inconsciente, além disso, tem algum tipo de identidade decorrentes dos arquétipos do Inconsciente Coletivo, que auxilia à formação de mitos e rituais, promovendo o desenvolvimento individual. (Henderson, 1990, p.103, tradução nossa)

Ao apresentar este conceito são dois os aspectos importantes a serem ressaltados: a localização do Inconsciente Cultural e a ênfase em uma área da memória histórica (Singer \& Kimbles, 2004a; Kimbles, 2006). A localização do Inconsciente Cultural se encontra entre as camadas individual e arquetípica, é o elo que permite a formação de mitos e ritos próprios de uma determinada cultura. O seu lócus é o grupo e o nível da psique do grupo. Com relação à ênfase na memória histórica, ela fornece uma espécie de continuidade viva entre passado e presente, ao nível inconsciente do grupo. Apesar da cultura e da história serem fundamentais na epistemologia junguiana e no desenvolvimento da personalidade (Vieira, 2004), não foram encontrados trabalhos que definam ou sistematizem de maneira rigorosa a noção de cultura em Jung, o mesmo ocorrendo no termo "Complexo Cultural".

Singer e Kimbles (2004b) ampliam algumas ideias de Henderson, entendendo que este conceito permite compreender a vida grupal como um 'tecido simbólico'. Ou seja, ele permite englobar e estruturar a dinâmica interna e externa, assim como a dimensão simbólica no âmbito das relações humanas, expressas em suas narrativas, interações e imagens. Uma "pele de grupo" é percebida então como "uma função contendo condensações coletivas, vulnerável a disseminações, rupturas, mortes e renovações. (...) a memória cultural (...) é um campo dinâmico e vivo" (Singer \& Kimbles, 2004b. p.184, tradução nossa).

Sobre a questão da presença da memória grupal e de seus símbolos, Kimbles (2014) comenta que as

memórias vivas internas no nível do inconsciente cultural são evidentes na representação que o grupo faz de si mesmo por meio do uso de símbolos coletivos embutidos em rituais de grupo, celebrações e narrativas sobre sua história. Esses processos criam um espaço simbólico cultural no qual memórias e eventos são realizados, elaborados e passam a significar o espírito do grupo. Os inúmeros rituais de comemoração, cerimônias e aniversários nacionais, celebrando tudo, desde a fundação do país, às vitórias da guerra, à nomeação de ruas e bibliotecas com nomes de heróis, são tentativas de estabelecer monumentos vivos para uma história que representa o grupo (p.69, tradução nossa). 
A acentuada ênfase das questões culturais, tanto nas contribuições de Henderson sobre o Inconsciente Cultural, quanto no alargamento destas ideias realizadas por Samuel Kimbles e Thomas Singer, demarcam esta área de intersecção entre o pessoal e o arquetípico. Ainda que Henderson tenha explorado esta lacuna do campo de estudos junguianos, o conceito de Inconsciente Cultural não propõe uma análise da dinâmica de eventos culturais, somente localiza esta dimensão da cultura na psique. É a partir da teoria dos complexos aplicada ao grupo que surge o conceito de Complexo Cultural como uma compreensão analítica dos fenômenos grupais.

\section{COMPLEXOS CULTURAIS}

\section{DEFINIÇÃO}

Complexo Cultural é um conceito com base na Psicologia Analítica e em diferentes vertentes deste campo teórico, apresentado com o propósito de estudar a dinâmica dos conflitos grupais. Podemos entendê-lo como componentes essenciais de uma sociologia interior, que não busca ser científica e objetiva em sua descrição de grupos e pessoas, visto que é filtrada por meio da psique de gerações de ancestrais (Singer \& Kimbles, 2004a).

Ele se estrutura, geralmente, a partir de vivências coletivas traumáticas relacionadas a temas históricos como: imigração, escravidão, colonização, conflitos entre estados e nações, relações étnicas e de gênero, religião, demarcação territorial, entre outros. Os diversos complexos locais podem estar presos em um mega complexo cultural.

Singer e Kimbles (2004a) entendem estes complexos como uma ampliação da teoria original de Jung. Para eles, é uma extensão do conceito de complexo ao nível grupal, demarcando a presença da dimensão grupal na psique individual. A própria utilização da terminologia "Complexo Cultural" é uma maneira de se referir aos seus conceitos predecessores, sendo também encontrado a utilização do termo "Complexo Grupal" (Kimbles, 2014; Singer, 2002, 2006a, 2006b; Singer \& Kimbles, 2004a).

Os complexos culturais possuem um funcionamento diferente dos complexos pessoais, ainda que compartilhem algumas características, como: autonomia, repetição, resistência a consciência, o acúmulo de experiências que confirmam seu ponto de vista histórico e que fornecem uma certeza simplista sobre o lugar do grupo no mundo (Singer 2002; Singer \& Kimbles, 2004b). Além disso, "os complexos pessoais e culturais possuem núcleos arquetípicos; isto é, eles expressam tipicamente atitudes humanas e estão enraizados em ideias primordiais sobre o que é importante, tornando-os muito difíceis de resistir, refletir e discriminar" (Singer, 2002; 2006a; 2006b). Contudo, os complexos culturais são mais amplos e, portanto, mais difíceis de serem trabalhados a nível do indivíduo. 
Estes núcleos afetivos grupais organizam a história psicológica de uma cultura, gerando um campo emocional próprio e autônomo, pertencendo não somente aos indivíduos, mas a própria cultura. Tanto as exigências do passado quanto os processos futuros são moldados por estes complexos. Eles permitem, assim, uma análise terapêutica cultural (Kimbles, 2003b).

Kimbles (2000) avalia os Complexos Culturais como uma "forma de entender o sistema dinâmico de relações que atendem a necessidade básica de pertença e identidade através da ligação de experiências pessoais e grupais" (p.160, tradução nossa). A base deste conceito se estrutura em seis aspectos desenvolvidos e ampliados por Kimbles (2000, 2003b, 2006, 2014), Singer (2003, 2006c, 2012c, 2013a, 2014a, 2016d, 2018a), Singer e Kimbles, (2004b) e Singer e Kaplinsky (2010) ao longo de suas produções, sendo:

1. Os Complexos Culturais são complexos que operam no nível do grupo do Inconsciente Cultural. Estes complexos organizam crenças e emoções coletivas profundamente arraigadas, organizando parte da vida em grupo. Eles mediam a relação entre indivíduo e o seu grupo de referência.

2. Funcionam de forma autônoma dentro de cada indivíduo ou grupo. A autonomia dos complexos sob a consciência se vincula à percepção restrita das diferenças. Enfatizando tanto a identificação e diferenciação do grupo, como o sentimento intenso de pertença e alienação dolorosa. Os Complexos Culturais são centros de nucleação em um movimento contínuo de imagens e afetos, levando a narrativas e rituais passados de geração em geração. Funcionam de maneira centrípeta, ou seja, por meio de uma força que puxa e mantém a psique grupal em si mesma, para o seu próprio centro.

3. Organizam as atitudes, emoções e comportamentos que compõem a vida em grupo. Os Complexos Culturais funcionam como campos emocionais, são impessoais e atuam em prol do grupo. Eles impulsionam em direção a sentimentos e ações, por meio de uma indução psíquica.

4. Facilitam a relação afetiva do indivíduo com os padrões culturais do grupo. Em seu aspecto positivo, estruturam o sentimento de pertencimento, sendo a identidade alcançada por meio da identificação com o grupo cultural, social ou étnico. Negativamente, geram preconceitos, atitude de ameaça a alteridade, dentro da dinâmica nós/eles (Us/Them) como categorias de longa data junto com imagens e respostas afetivas estereotipadas.

5. Proporcionam ao indivíduo e ao grupo um senso de pertencimento e identidade dentro de uma continuidade histórica de pressupostos emocionais compartilhados. Este senso 
de pertencimento se vincula tanto aos eventos negativos (linchamento de multidões), quanto positivos (patriotismo).

6. São uma maneira da psique de narrar sua relação com o grupo. Os Complexos Culturais incorporam elementos de mitologia, política e psicologia, como uma sociologia interna composta também por um pouco de história, economia, antropologia e poesia. A perspectiva junguiana, aliada a estas outras áreas do conhecimento, privilegia a base mitopoética da psique e a sua realidade psíquica. Muito mais que um olhar objetivo para os seus constructos, são exploradas as relações a partir do mundo interior.

Diferentes questões sociais podem ser sustentadas por Complexos Culturais, como: relações de classe, desemprego permanente, disparidade na educação, sistema de justiça criminal, divisão familiar e desordem social. Estas questões trazem consigo intensa carga afetiva, exigindo mudança no nível da fantasia.

A nível social, diferentes palavras podem constelar Complexos Culturais, vinculados a identificação política ou ideológica, mobilizando fantasias, ideias, pensamentos e afetos. Após o atentado de 11 de setembro, palavras como "eixo do mal", "inimigo", "guerra santa", "terrorismo global", passaram a produzir uma linguagem de sinais emocionais coletivos entre os estadunidenses. Reduzindo-se em uma batalha nós/eles (Us/Them), cegando a população em busca de justiça (Kimbles, 2003b).

Ainda que possam ter alguns elementos semelhantes, Complexo Cultural não é o mesmo que Identidade Cultural ou Caráter Nacional. Mesmo que um Complexo Cultural possa ser assumido como uma Identidade Cultural 'saudável' (Ego Cultural) de indivíduos e grupos, ele não se reduz a isso, podendo irromper a partir de seu polo mais danoso, que fragmenta a psique grupal. Enquanto isso, a Identidade Cultural está mais vinculada com as noções e características de determinado grupo ou nação. A noção de Identidade Cultural na discussão sobre Caráter Nacional foi um ponto controverso na teoria junguiana, sendo vinculado a uma ideia antissemita. A constelação de um Complexo Cultural com alta carga afetiva reduz as percepções de um grupo, a uma relação unilateral e dicotômica. Em contrapartida, um indivíduo ou grupo que possua uma Identidade Cultural própria possui maior liberdade na interação com outros grupos, destituindo-se de amarras emocionais que restrinjam estas relações a conflitos intergrupais.

A partir disso, podemos pensar que os Complexos Culturais se constroem como uma maneira da psique narrar a sua relação com o grupo, por meio de dinâmicas inconscientes da vida grupal, vinculando sentimento de pertencimento e expectativas grupais a experiências pessoais, funcionando, assim, como um pano de fundo emocional. Ainda que, "a sociologia interna dos Complexos Culturais pode aproveitar a imaginação, o comportamento e as emoções da psique coletiva e desencadear forças tremendamente irracionais em nome de sua 'lógica'" (Singer e Kimbles, 2004a, tradução nossa). 
A descrição e análise de um Complexo Cultural não possui metodologia definida, mas podem ter alguns elementos norteadores. Singer (2018a) identifica características similares à metodologia de pesquisa nas ciências sociais, baseado nos conceitos de informantes-chaves, como forma de coletar evidências qualitativas na investigação de Complexos Culturais, somado à uma base mitopoética da psique. Ademais, algumas perguntas podem servir de base para identificar e descrever um Complexo Cultural, como: que sentimentos estão vinculados a este complexo?; que imagens aparecem?; que memórias se constelam quando se apresenta este complexo?; que comportamentos são desencadeados?; e, que pensamentos estereotipados se repetem? (Singer, 2018a).

Para além da definição e metodologia, questões como a cura e resolução de um Complexo Cultural foram uma das preocupações de Singer e Kimbles. A seguir, discutiremos sobre a função transcendente na sociedade, e o processo de integração envolvido como uma forma de "cura" dos Complexos Culturais.

\section{INTEGRAÇÃO DOS COMPLEXOS CULTURAIS}

Ainda que a proposta dos Complexos Culturais não objetive uma cura, diferentes ideias neste sentido têm sido apresentadas. Se concebe que uma real cura implica em um esforço coletivo, e não somente um esforço individual na assimilação desses traumas sociais/psicológicos, tanto dos perpetradores e dos benificiários silenciosos, como das vítimas (Kimbles, 2000; Singer, 2003).

São sugeridos três pontos para uma possível cura dos Complexos Culturais:

[1] eles podem ser resolvidos como o curso de uma febre. (...) é uma questão de tempo para que as defesas arquetípicas do espírito coletivo percam a sua energia psíquica e voltem ao inconsciente cultural. (...) [2] Em outras raras ocasiões, no surgimento de um líder carismático que exerça a função transcendente na psique coletiva [como o exemplo de] (...) Gandhi, Martin Luther King, Nelson Mandela ou Barack Obama. (...) [3] Alguns indivíduos talentosos podem ter a capacidade de vivenciar conscientemente os complexos culturais que dividem os grupos em facções guerreiras. Eles têm a capacidade de manter esses complexos culturais em sua psique de tal forma que uma experiência transcendente autêntica ganha vida neles, apontando para uma resolução de outra forma intratável, como os conflitos grupais afetivos. (Singer, 2012b, p. 23, tradução nossa).

Essas possíveis curas operam a partir da função transcendente na sociedade. A função transcendente, como entendida por Jung (1958/2013b), acontece na relação que se estabelece entre consciente e inconsciente, tendo como mediador desta relação o símbolo. A função transcendente produz a tensão energética entre os opostos, e, com a formação do símbolo, algo vivo, uma nova situação psíquica. 
A função transcendente na sociedade é uma ampliação de Singer (2010, 2012b) do conceito de Jung para uma dimensão grupal e coletiva.

A função transcendente na sociedade acontece tanto como um evento momentâneo quanto como um processo ao longo do tempo, podendo suceder no coletivo por meio de um indivíduo excepcional ou por um grupo de cidadãos comuns. Ela pode trazer encorajamento e ajuda a nível coletivo para que um Complexo Cultural cesse ou seja mitigado. Alguns exemplos desses processos coletivos são: a proclamação da assunção da Virgem Maria, relacionado a um Complexo Cultural da relação entre mulheres e homens; um discurso sobre raça de Barack Obama, que reconhece a diferente racial na sociedade estadunidense e profere esta fala de maneira mediadora entre brancos e negros; e a Proposition 8 e o filme Milk, ambos versam sobre a temática da homossexualidade. Eles sinalizam que a função transcendente na sociedade pode ocorrer como um processo ao longo do tempo, já que a Proposition 8 foi uma iniciativa que buscava banir o casamento homoafetivo na Califórnia, e, simultâneo a isso, estreou o filme Milk, que retratou a vida de Harvey Milk, o primeiro homem declaradamente homossexual a ser eleito para um cargo público na California. Esta ambivalência denota justamente como os Complexos Culturais podem se manifestar de maneira progressivo e regressivo na psique coletiva. (Singer, 2010, Singer, 2012b).

Ainda que Singer (2010) tenha focado as suas análises partindo de uma perspectiva psicológica, comentamos que esses exemplos poderiam ter sido avaliados também a partir de seus aspectos políticos e ideológicos, entendendo estes como unidades de análise que poderiam ampliar os seus estudos.

Ainda sobre a função transcendente, Singer (2012b) expõe seu entendimento desse processo a nível coletivo:

não creio que a "função transcendente" por si só faça com que os Complexos Culturais se resolvam, como uma intervenção mágica ou divina, que inverte o curso da história. Sua aparência é introduzida apenas pelo trabalho duro, enorme sacrifício e profundo sofrimento de muitos indivíduos e grupos que foram vítimas de Complexos Culturais, de pessoas que levantaram suas vozes em protesto contra essas injustiças. Tal "testemunho" de indivíduos e grupos pode trazer à existência a função transcendente que pode, por sua vez, sinalizar uma mudança no curso de potentes Complexos Culturais com histórias multigeracionais. Podemos "sentir" a atividade da "função transcendente" ao acordarmos para o fato de que ocorreu uma mudança profunda na psique coletiva que é tão real quanto inesperada (p. 24, tradução nossa).

No painel 'The transcendente function in society', apresentado por Meador, Samuels \& Singer (2010), Andrew Samuels afirmou a sua não concordância com a utilização do conceito de 'função transcendente na sociedade'. Para isso, utiliza um exemplo 
sobre racismo, se questionando em como um grupo com ideias racista e outro com ideias não racistas poderiam promover uma função transcendente. Ou seja, qual seria esse terceiro elemento que emergiriam desta relação, produto deste par de opostos, pessoas "semi-racistas"?

Samuels retoma também algumas ideias apresentadas em seu livro 'A Psique Política', entendendo que as ferramentas conceituais para uma análise social/cultural/política devem passar por um exame criterioso. Ademais, devesse ter cautela na aplicação de conceitos clínicos para se realizar tal análise.

Se pensarmos em uma das formas de cura de um Complexo Cultural como o seu retorno ao Inconsciente Cultural após a perda de energia psíquica, seria possível classificar tal fato como cura ou poderíamos pensar melhor como mitigação deste complexo? A nível pessoal, a simples perda da energia de um complexo não pressupõe uma nova forma de relação com ele, mas expressa a diminuição de sua autonomia na psique.

Segundo o dicionário crítico de análise junguiana, a análise pode prover algo parecido com a 'cura', ainda que não haja qualquer forma de psicoterapia que possa efetuar a 'cura' (Samuels, Shorter, \& Plaut, 1998). Jung (1949/2012) comenta que a cura de uma neurose implica em uma relação responsável de conscientização, entre as partes. Em última análise, uma neurose implica em um problema moral.

Assim, falar em um processo de cura envolve relação, conscientização de aspectos inconscientes, traumas. A nível pessoal é um processo que pode se prolongar por anos. Como então pensar em cura quando falamos em Complexos Culturais? Propõe-se entender a cura de um Complexo Cultural mais como a abertura de uma nova forma de relação, a nível individual e grupal, com as questões pertinentes ao grupo. A figura de um líder carismático pode facilitar, fortalecer, dar continência para que conteúdos possam emergir, mantendo-os presentes na psique coletiva.

Por fim, diferente de Thomas Singer (2010, 2012b), que utilizou o termo "cura", sugerimos a utilização do termo integração dos Complexos Culturais, condizente com a teoria junguiana, já que não há uma "cura" para os complexos. A integração se caracteriza pela assimilação parcial de conteúdos inconscientes, o que denota uma relação dinâmica entre consciente e inconsciente.

\section{DERIVAÇÕES CONCEITUAIS E A IMPLICAÇÃO DOS COMPLEXOS CULTURAIS NA PRÁTICA CLÍNICA}

Um aspecto pouco estudado é a ideia de finalidade ou o telos de um Complexo Cultural. Jung (1928/2013) entende que os fenômenos psicológicos devem ser abordados a partir de um duplo ponto de vista, a saber: regressivo e causal ou progressivo e finalista. O autor privilegia o sentido finalista e entende que esta perspectiva tem "um objetivo a se alcançar" (§ 456). Na psique pessoal esta finalidade se organiza a partir do centro autorregulador da psique, o Si-mesmo. 
Singer (2016b), ao escrever sobre o Obamacare enquanto Complexo Cultural, apresenta uma contribuição de John Beebe, em uma comunicação oral, sobre a necessidade de se pensar a partir da ideia de telos. A finalidade de um complexo se refere justamente as suas questões que permanecem paralisadas. $O$ telos do Obamacare:

é forçar-nos a olhar para o Darwinismo Social em ação, de modo a nos fazer ver quão inadequada essa ou qualquer outra teoria pode ser quando convocada para impedir uma investigação mais profunda da afirmação da alma sobre as questões que envolvem um assunto tão grave quanto uma sociedade propõe cuidar dos cidadãos que adoecem (Beebe, 2015 citado por Singer, 2016b. p.153, tradução nossa).

Ainda que esta seja uma proposição breve e pouco discutida por Singer em outros textos, pensar no telos parece ser uma ferramenta válida para se entender o propósito e finalidade de um Complexo Cultural.

Neste ponto, podemos retomar uma afirmação feita por Singer (2003), sobre a necessidade de se respeitar as diferentes camadas da psique (pessoal, cultural e arquetípica), sem sobrepor ou valorizar uma em detrimento da outra. A questão é: ainda que o Complexo Cultural pode, eventualmente, se entrelaçar a um complexo individual, se cumpriria uma única finalidade? Ou seja, a nível coletivo ele pode cumprir uma função e a nível pessoal pode cumprir outra? Se este for o caso, caberia uma análise coletiva e outra individual, singularizada.

Outra implicação dos Complexos Culturais é apresentada no pensamento da prática clínica junguiana. Estes complexos estão relacionados aos complexos pessoais, de alguma maneira, podendo ser percebidos nos sonhos e fantasias do paciente ou ainda, na transferência/contratransferência (Kimbles, 2004). Kimbles (2000) discorre mais sobre o tema em outro artigo:

Ao longo dos anos, notei com frequência em situações clínicas que quando os sentimentos profundos sobre etnia, raça e gênero são afetados, eles geralmente mudam a maneira como eu e o paciente experienciamos um ao outro. Nós dois parecemos apanhados por algo pegajoso, emocional, organizado dentro de um padrão de sentimentos que envolvem aspectos de 'dentro do grupo'/'fora do grupo' (ou nós/eles). Esses sentimentos conectam cada um de nós, por meio de uma narrativa social, aos nossos diferentes grupos de referência. Isso pode ocasionar projeção, culpa e mal-entendidos que devem ser analisados ( $p$. 159, tradução nossa)

De fato, a influência de questões étnicas e culturais devem permear também a análise junguiana. Para Kimbles (2004), em seu viés mais fragmentado, o papel das diferenças pode gerar aspectos como culpa, agressão e negação, tendo estes 
complexos a possibilidade de gerar excessiva ambivalência, curiosidade, dúvida, defesa e confusão.

Assim como na prática clínica junguiana existe um arcabouço conceitual que norteia o trabalho do psicólogo, ao longo dos anos, Samuel Kimbles e Thomas Singer têm agregado diferentes conceitos no estudo dos Complexos Culturais. A medida em que os estudos neste tema avançaram, alguns conceitos foram utilizados como complementares em suas análises. Especulamos que a sua finalidade foi dar maior consistência nos estudos relacionados a transmissão intergeracional, a mobilização psíquica do grupo referente a traumas coletivos, ou ainda, na projeção da sombra intergrupal.

O foco de Samuel Kimbles nos estudos sobre Complexo Cultural são temas vinculados ao racismo e trauma transgeracional/intergeracional. O autor propõe conceitos como Narrativas Fantasmas, Processo de Sombra Coletiva, Sofrimento social por meio do Luto Cultural e da Melancolia Cultural. Enquanto os Complexos Culturais passam a organizar as relações inter e intragrupais, conceitos como Narrativas Fantasmas atuam, por meio desses complexos, na manutenção de narrativas inconscientes no grupo. Enquanto o conceito de sofrimento social, apresentado por meio do luto e melancolia cultural, contribui na percepção dos eventos sociais traumáticos perpetuados no cotidiano, principalmente relacionados a pobreza e seus desdobramentos na psique de populações que vivem em situação de vulnerabilidade social.

Os estudos de Thomas Singer estão relacionados a temas como política narcisismo, ansiedade de extinção e o governo de Donald Trump (Singer, 2013b, 2016a, 2016b, 2017, 2018a, 2018b), o Obamacare como um Complexo Cultural (Singer, 2016b), a função transcendente na sociedade, bem como, a vivência de traumas culturais a nível coletivo, nomeado como as 'defesas arquetípicas do espírito coletivo'. Estas defesas são entendidas como a evocação dos Daimones para a preservação da psique grupal, como expresso no mito do bebê Zeus e os Curetes.

Um dos exemplos da apresentação da dinâmica das defesas arquetípicas do espírito coletivo está no primeiro texto sobre Complexo Cultural de Singer (2002), sobre Elián Gonzales. Este é o caso de um menino cubano levado pela mãe aos Estados Unidos e que desperta e mobiliza tanto a comunidade cubanoamerica quanto os cubanos. A primeira querendo manter o garoto em solo estadunidense e a última querendo o retorno dele a família, em Cuba. Singer (2002) entendeu este evento como a expressão das defesas arquetípicas do espírito coletivo expressos no grupo cubanoamericano, que buscava proteger o jovem Elián.

Graaff (2018) propõe uma reinterpretação deste evento, criticando a abordagem de Singer (2002) e a escassa utilização de dados históricos em sua análise sobre o caso Elián Gonzales, bem como do uso do conceito de defesas arquetípicas do espírito coletivo para explicá-lo. Ao retomar a história deste caso, Graaff (2018) apresenta outras imagens possíveis para análise como a Madona Negra e não 
somente a imagem de uma criança divina sendo raptada de sua terra natal, como propôs Singer (2002). Este trabalho apresenta diferentes níveis em sua análise, como o pessoal, familiar e comunitário, descrevendo-as de maneira separada.

Kevin Lu (2013a; 2013b) também expõe algumas críticas à teoria dos Complexos Culturais, como o não reconhecimento de outras obras, dentro da psicologia profunda, que realizam análises sociais. Comenta também sobre o perigo de se transpor conceitos clínicos para se realizar análises culturais, preocupação pontuada também por Samuels (2010). Além disso, Lu (2013a) se preocupa com a interlocução deste conceito com outras áreas, criticando a utilização inapropriada de disciplinas como a história, afirmando a ausência do uso rigoroso de critérios de verificação utilizado pelos historiadores.

Em resposta a Lu, Singer (2013a) afirma que a sua perspectiva de análise parte de uma ferramenta bem estabelecida, utilizada no ARAS (Archive for Research in Archetypal Symbolism). Comenta também que esta não busca ser a única proposta de análise cultural, algo como uma teoria junguiana que unifique as análises do coletivo. Ademais, comenta que a preocupação da teoria dos Complexos Culturais busca trabalhar com a realidade psíquica, entendendo esta como a principal contribuição deste conceito.

De modo geral, algumas dessas críticas são pertinentes na medida em que auxiliam na qualificação do conceito dos Complexos Culturais. Além disso, chamam a atenção para a necessidade de maior rigor, tanto dentro do próprio campo junguiano, pensando nos conceitos propostos neste marco teórico, quanto no amparo em outras áreas do conhecimento.

\section{CONCLUSÃO}

Este artigo se propôs a analisar conceitualmente a teoria dos Complexos Culturais, tendo em vista o expressivo número de publicações sobre este recente conceito junguiano, bem como a inexistência de estudos que empreenderam tal proposta anteriormente. Como apresentado, a busca de consistência em um corpo teórico requer a revisão de seus conceitos fundantes.

Os Complexos Culturais têm sido utilizados como ferramenta importante para se descrever a dinâmica dos conflitos grupais, ampliando a perspectiva de estudo e possibilidade de análise dos fenômenos culturais e grupais. A partir deste marco teórico, os complexos têm presença na psique grupal e no nível grupal da psique individual. Eles organizam as relações do indivíduo com o grupo por meio de imagens e ideias com um núcleo afetivo. Proporcionam uma sensação de pertencimento e identidade.

Apresentou-se as concepções de Jung em relação ao coletivo. No léxico junguiano, a palavra coletivo possui diferentes significados, como psique coletiva, Inconsciente Coletivo, ou ainda, pode estar relacionado ao conceito de massa. Jung 
deixou uma lacuna nos estudos sobre cultura e as suas relações com a psique. A partir disso, Henderson (1990) propôs o conceito de Inconsciente Cultural, entendendo esta como uma camada da psique entre o Inconsciente Pessoal e o Inconsciente Coletivo.

Algumas críticas à incursão dos junguianos no estudo cultural e de grupos (Graaff, 2018; Lu, 2013a, 2013b), são pontos válidos para se pensar em critérios para uma análise cultural junguiana, seja pelo perigo de se realizar apenas uma transposição e aplicação da noção clínica para o social, ou ainda, o risco de se realizar uma 'psicanálise selvagem'. O intercâmbio de ideias com outras áreas exige rigor e pede avaliação do alcance e/ou limitações da sua utilização.

Ao selecionar as obras dos dois principais autores sobre o conceito, percebeu-se a utilização de algumas derivações conceituais. Samuel Kimbles possui estudos vinculados ao racismo e trauma transgeracional/intergeracional. O autor propõe alguns conceitos complementares que buscam dar maior consistência para articulação com os seus assuntos de interesse, como: Narrativas Fantasmas, Processo de Sombra Coletiva, Sofrimento social por meio do Luto Cultural e da Melancolia Cultural. Enquanto Thomas Singer, apresenta estudos relacionados a dinâmica do trauma coletivo - defesas arquetípicas do espírito coletivo -, além de análises relacionadas a política estadunidense e possibilidades de cura dos Complexos Culturais.

Demarcar um conceito, verificar os seus avanços ao longo do tempo e perceber o seu alcance foram alguns dos objetivos deste artigo. Os Complexos Culturais têm ocupado um lugar importante no campo junguiano para se analisar a constante transição da civilização. Além disso, têm se mostrado um dos principais conceitos de estudos dos teóricos junguianos em análises sociais.

\section{REFERÊNCIAS}

Adams, M. (1996). The cultural unconscious and the collective differences. In The multicultural imagination: "Race", Color and the Unconscious. (pp. 37-50). New York: Routledge.

Araujo, F. C. (2002). Da cultura ao inconsciente cultural: psicologia e diversidade étnica no Brasil contemporâneo. Psicologia: ciência e profissão, 22(4), 24-33. Recuperado de http://pepsic.bvsalud.org/scielo.php?script=sci_arttext\&pid=S141498932002000400004\&lng=pt\&tlng=pt.

Boechat, W. (2009, setembro). Eros, poder e o racismo cordial: aspectos da formação da identidade brasileira. Anais do V Congreso Latinoamericano de Psicología Junguiana. Santiago, Chile.

Boechat, W. (2012). Cordial Racism: Race as a Cultural Complex. In P. Amezaga, G. Barcellos A. Capriles, J. Gerson, D. Ramos (Ed.), Listening to Latin America: Exploring cultural complexes in Brazil, Chile, Colombia, Mexico, Uruguay, and Venezuela. (pp. 31-50). New Orleans: Spring Journal Books.

Boechat, W. (Ed.). (2014). A alma brasileira: luzes e sombra. Petrópolis: Vozes.

Boechat, W. (2016). Racism: an unwelcome guest in Brazilian cultural identity. In E. Kiehl, M. Saban, A. Samuels (Ed.), Analysis and Activism: Social and Political Contributions of Jungian Psychology. (pp. 125-131). New York: Routledge.

Boechat, (2018). Cultural complexes and cultural identity in Brazil: the development of an individual identity. In J. Cambray, L. Sawin. (Ed.), Research in Analytical Psychology: Applications from Scientific, Historical, and Cross-Cultural Research: Volume 1. New York: Routledge.

Dias, L \& Gambini, R. (1999). Outros 500: uma conversa sobra a alma brasileira. São Paulo: Senac. 
Gambini, R. (2000). O espelho índio: A formação da alma brasileira. São Paulo: Axis mundi: Terceiro Nome.

Gambini, R. (2003). Soul and Culture. Texas: Texas A\&M University Press.

Geertz, C. (1973/2008). A interpretação das culturas. Rio de Janeiro: LTC.

Graaff, J. (2018). Re-interpreting Elián González: The Puzzles of the Cultural Complex. Jung Journal: Culture \& Psyche, 12(1), 42-56. https://doi.org/10.1080/19342039.2018.1403255

Henderson, J. (1984). Cultural Attitudes in Psychological Perspective. Toronto: Inner City Books.

Henderson, J. (1990). The Cultural Unconscious. In J. Henderson. Shadow and Self: Selected Papers in Analytical Psychology. (pp. 103-113). Chicago: Chiron.

Hillman, J. (1986). Notes on White supremacy: Essaying an archetypal account of historical events. Spring, 29-58

Jung, C. (1902/2013). Sobre a psicologia e patologia dos fenômenos chamados ocultos. In Estudos psiquiátricos. (pp. 11-103). Petrópolis: Vozes. (Obras Completas v. 1).

Jung, C. (1903/2013). Sobre a estimulação de distúrbio mental. In Estudos psiquiátricos. (pp.167-196). Petrópolis: Vozes. (Obras Completas v. 1).

Jung, C. (1904/2013). Parecer médico sobre um caso de simulação de insanidade mental. In Estudos psiquiátricos. (pp.197-214). Petrópolis: Vozes. (Obras Completas v. 1).

Jung, C. (1904/2012). Estudos diagnósticos de associações. In Estudos Experimentais. (pp. 9-248). Petrópolis: Vozes. (Obras Completas, volume 2).

Jung, C. (1905/2013). Criptomnésia. In Estudos psiquiátricos. (pp.108-119). Petrópolis: Vozes. (Obras Completas v. 1).

Jung, C. (1916/2012). Adaptação, individuação e coletividade. In A vida simbólica. (pp. 205-211). Petrópolis: Vozes. (Obras completas, v. 18/2).

Jung, C. (1916/2015). O eu e o inconsciente. Petrópolis: Vozes. (Obras Completas, v. 7/2).

Jung, C. (1919/2013). Instinto e Inconsciente. In A natureza da psique. (pp. 72-82). Petrópolis: Vozes. (Obras Completas v. 8/2).

Jung, C. (1920/2013). Tipos Psicológicos. (pp. 467-468). Petrópolis: Vozes. (Obras Completas, volume 6).

Jung, C. (1925/2014). Seminários sobre psicologia analítica (1925). Petrópolis: Vozes.

Jung, C. (1928/2013). Aspectos gerais da psicologia do sonho. In A natureza da psique. (pp. 186-234). Petrópolis: Vozes. (Obras Completas v. 8/2).

Jung, C. (1934/2013). Considerações gerais sobre a teoria dos complexos. In A natureza da psique. (pp. 39-52). Petrópolis: Vozes. (Obras Completas v. 8/2).

Jung, C. (1936/2012). Wotan. In Aspectos do drama contemporâneo. (pp. 13-27). Petrópolis: Vozes. (Obras completas, v. 10/2)

Jung, C. (1949/2012). Prefácio ao livro de Neumann "Depth psychology and a new ethic". In $A$ vida simbólica. (pp. 205-211). Petrópolis: Vozes. (Obras completas, v. 18/2).

Jung, C. (1950/2013). Símbolos da transformação. Petrópolis: Vozes. (Obras Completas, v. 5).

Jung, C. (1953/2013). Um mito moderno sobre as coisas vistas no céu. Petrópolis: Vozes. (Obras completas, v. 10/4).

Jung, C. (1958/2013a). Presente e futuro. Petrópolis: Vozes. (Obras completas, v. 10/1).

Jung, C. (1958/2013b). A função transcendente. In A natureza da psique. (pp.13-38). Petrópolis: Vozes. (Obras Completas v. 8/2).

Kimbles, S. (1998). Panacea and poison in analytic training. In M. Mattoon (Ed.), Florence 1998 Destruction and Creation: Personal and Cultural Transformations. (pp. 440-445). Einsiedeln: Daimon.

Kimbles, S. (2000). The Cultural Complex and the Myth of Invisibility. In T. Singer. (Ed.), The Vision Thing. (pp. 157-169). New York: Routledge.

Kimbles, S. (2003a). Joe Henderson and the Cultural Unconscious. The San Francisco Jung Institute Library Journal. 22(2), 53-58. https://doi.org/10.1525/jung.1.2003.22.2.53

Kimbles, S. (2003b). Cultural complexes and collective shadow processes. In J. Beebe. (Ed.), Terror, violence and the impulse to destroy. (pp. 211-33). Einsiedeln, Switzerland: Daimon Press.

Kimbles, S. (2004). A cultural complex operating in the overlap of clinical and cultural space. In T. Singer, S. Kimbles. (Eds.), The cultural complex: Contemporary Jungian perspectives on psyche and society. New York: Routledge.

Kimbles, S. (2006). Cultural complexes and the transmission of group traumas in everyday life. Psychology Perspectives, 49(1), 96-110. 
Kimbles, S. (2007). Social Suffering through Cultural Mourning, Cultural Melancholia, and Cultural Complexes. Spring: A Journal of Archetype and Culture. Vol. 78.

Kimbles, S. (2014). Phantom narratives: The unseen contributions of culture to psyche. Lanham, MD: Rowman \& Littlefield.

Kimbles, S. (2016). Phantom narratives and the uncanny in cultural life: psychic presences and their shadows. European Journal of Psychotherapy \& Counselling, 18(2), 156-169. https://doi.org/10.1080/13642537.2016.1170061

Kimbles, S. (2017). Floating worlds and their phantoms in the aftermath of social catastrophes. J Anal Psychol, 62, 130-146. doi: 10.1111/1468-5922.12289.

Laurenti, C. \& Lopes, C. (2016). Metodologia da pesquisa conceitual em psicologia. In C. Laurenti, C. Lopes, S. Araujo. (Eds.), Pesquisa Teórica em Psicologia. São Paulo: Hogrefe.

Lu, K. (2013a). Can individual psychology explain social phenomena? An appraisal of the theory of cultural complexes. Psychoanalysis, Culture \& Society, 18(4), 386-404. https://doi.org/10.1057/pcs.2012.43

Lu, K. (2013b). Reply to Thomas Singer. Psychoanalysis, Culture \& Society, 18(4), 416-422. https://doi.org/10.1057/pcs.2012.43

Machado, A., Lourenço, O., \& Silva, F. (2000). Facts, concepts, and theories: the shape of psychology's epistemic triangle. Behavior and Philosophy, Cambridge, 28, 1-40.

Novaes, C. (2016). Corrupção no Brasil: uma visão da Psicologia Analítica. Junguiana, 34(2), 5-17.

Odajynk, V. (1976/2007). Jung and politics: The political and social ideas of C. G. Jung. New York: Harper.

Oliveira, H. (2018). Desvelando a alma brasileira: Psicologia junguiana e raízes culturais. Petrópolis: Vozes.

Oliveira, H. (2020). Morte e renascimento da ancestralidade indígena na alma brasileira: psicologia junguiana e inconsciente cultural. Petrópolis: Vozes.

Progoff, I. (1953). Jung's psychology and its social meaning; An introductory statement of C.G. Jung's psychological theories and a first interpretation of their significance for the social sciences. New York: Grove Press.

Ramos, D. (2004). Corruption: Symptom of a cultural complex in Brazil? In T. Singer, S. Kimbles (Eds.) The Cultural Complex: Contemporary Jungian perspectives on psyche and society. (pp. 102123). New York: Routledge.

Ramos, D. (2010). Creativity and art as part of the elaboration of trauma brought on by slavery. In M. Stein, R. Jones (Ed.), Cultures and Identities in Transition: Jungian Perspectives. New York: Routledge.

Ramos, D. (2011). Le complexe culturel et l'élaboration du traumatisme de l'esclavage. Cahiers jungiens de psychanalyse, 133(1). 59-77.

Ramos, D. (2012). Cultural Complex and the Elaboration of Trauma of Slavery. In P. Bennett (Ed.) Montreal 2010-Facing Multiplicity: Psyche, Nature, Culture: Proceedings of the 18th Congress of the International Association for Analytical Psychology. Einsiedeln, Switzerland: Daimon.

Samuels, A. (1989/1992). A psique plural. Rio de Janeiro: Imago.

Samuels, A. (1993/1995). A psique política. Rio de Janeiro: Imago.

Samuels, A. (2001/2002). A política no divã. São Paulo: Summus.

Samuels, A. (2010). The transcendent function and politics: NO! In B. Meador A. Samuels, T. Singer. The transcendent function in society. Journal of Analytical Psychology. 55(2), 228-253.

Samuels, A., Shorter, B. Plaut, F. (1998). Dicionário crítico de análise junguiana. Rio de Janeiro: Imago/Consultoria Editora.

Singer, T. (2002). The Cultural Complex and Archetypal Defenses of the Collective Spirit: Baby Zeus, Elian Gonzales, Constantine's Sword, and Other Holy Wars. San Francisco Jung Institute Library Journal, 20(4), 4-28.

Singer, T. (2003). Cultural Complexes and Archetypal Defenses of the Spirit. In J. Beebe (Ed.), Terror, violence and the impulse to destroy. (pp. 191-210). Einsiedeln, Switzerland: Daimon Press.

Singer, T. (2006a). The cultural complex: a statement of the theory and its application. Psychother. Politics Int., 4, 197-212. doi:10.1002/ppi.110

Singer, T. (2006b). Unconscious Forces Shaping International Conflicts: Archetypal Defenses of the Group Spirit from Revolutionary America to Confrontation in the Middle East. The San Francisco Jung Institute Library Journal, 25(4), 6-28. https://doi.org/10.1525/jung.1.2006.25.4.6 
Singer, T. (2006c). The Cultural Complex in Theory and Practice. In L. Cowan (Ed.), Barcelona 2004 Edges of Experience: Memory and Emergence. Proceeding of the Sixteeth International Conference for Analytical Psychology. Einsiedeln, Switzerland: Daimon.

Singer, T. (2007). A Personal Reflection on Politics and the American Soul. Spring: A Journal of Archetype and Culture. Vol. 78

Singer, T. (2009). A Jungian approach to understanding 'us vs them' dynamics. Psychoanalysis, Culture and Society, 14(1), 32-40.

Singer, T. (2010). The transcendent function and cultural complexes: a working hypothesis. In B. Meador A. Samuels, T. Singer. The transcendent function in society. Journal of Analytical Psychology. 55(2), 228-253.

Singer, T. (2012a). The Meshugana Complex: Notes from a Big Galoot Galut. Jung Journal: Culture \& Psyche, 6(1), 72-84. https://doi.org/10.1525/jung.2012.6.1.72

Singer, T. (2012b). The transcendent function, cultural complexes and change in society. Irish Journal of Counselling and Psychotherapy, 12(2), 22-26.

Singer, T. (2012c). Introduction. In P. Amezaga., G. Barcellos, A. Capriles, J. Gerson, D. Ramos (Eds.), Listening to Latin America: Exploring cultural complexes in Brazil, Chile, Colombia, Mexico, Uruguay, and Venezuela. (pp. 1-13). New Orleans: Spring Journal Books.

Singer, T. (2013a). Response to Kevin Lu's "Can individual psychology explain social phenomena? An appraisal of the theory of cultural complexes." Psychoanalysis, Culture \& Society, 18(4), 405415. https://doi.org/10.1057/pcs.2013.18

Singer. T. (2013b). Playing the race card: A cultural complex in action. In G. Heuer (Ed.), Sacral Revolutions Reflecting on the Work of Andrew Samuels - Cutting Edges in Psychoanalysis and Jungian Analysis. New York: Routledge

Singer, T. (2014a). On the Cultural Complex: Introductory Remarks to a 2013 IAAP Copenhagen Conference Panel. In E. Kiehl (Ed.), Copenhagen 2013 - 100 Years On: Origins, Innovations and Controversies. Einsiedeln, Switzerland: Daimon.

Singer, T. (2014b). Psyche and Society: Some Personal Reflections on the Development of the Cultural Complex Theory. Journal of Jungian Scholarly Studies, 9(1), 1-12. Recuperado de http://www.jungiansociety.org/images/e-journal/Volume-9/JSSSvol9-2014.pdf\#page=7

Singer, T. (2015). Is America's "Money Complex" bankrupting Its Character? In P. Peay (Ed.), America on the Couch: Psychological Perspectives on American Politics and Culture. (pp. 312-323). Brooklyn: Lantern.

Singer, T. (2016a). Trump and the American Selfie Spirit. In S. Carta, A. Adorisio, R. Mercurio (Eds.), The Analyst in the Polis (Volume I). Rome: Street Lib.

Singer, T. (2016b). Snapshots of the obamacare cultural complex. In E. Kiehl, M. Saban, A. Samuels (Eds.), Analysis and Activism: Social and Political Contributions of Jungian Psychology (pp. 147-155). New York: Routledge.

Singer, T. (2016c). President Trump and the American Selfie Spirit: Archetypal Defenses of the Group Spirit. In S. Busen, L. Cruz, J. Bolen (Eds.), A Clear and Present Danger: Narcissism in the Era of President Trump. Asheville: Chiron

Singer, T. (2016d). Introduction. In T. Singer, J. Rasche (Eds.), Europe's Many Souls: Exploring Cultural Complexes and Identities. (pp. 1-13). New Orleans: Spring Journal Books.

Singer, T. (2017). Donald Trump and the American Collective Psyche. In B. Lee (Ed.), The Dangerous Case of Donald Trump: 27 Psychiatrists and Mental Health Experts Assess a President. New York: St. Martin's Press.

Singer, T. (2018a). The cultural complex theory: scientific and mythopoetic ways of knowing. In J. Cambray, L. Sawin. (Eds.), Research in Analytical Psychology: Applications from Scientific, Historical, and Cross-Cultural Research: Volume 1. New York: Routledge.

Singer, T. (2018b). Extinction Anxiety and Donald Trump. In S. Buser, J. Gartner (Eds.), Rocket Man: Nuclear Madness and the Mind of Donald Trump. Asheville: Chiron.

Singer, T. \& Kaplinsky, C. (2010). Cultural Complexes in Analysis. In M. Stein (Ed.), Jungian Psychoanalysis: Working in the Spirit of C.G. Jung (pp. 22-37). Open Court Publishing Company: Chicago.

Singer, T. \& Kimbles, S. (Eds.). (2004a). The cultural complex: Contemporary Jungian perspectives on psyche and society. New York: Routledge.

Singer, T. \& Kimbles, S. (2004b). The emerging theory of cultural complexes. In J. Cambray, L. Carter (Eds.), Analytical psychology: Contemporary perspectives in Jungian analysis. (pp. 176-203). New York: Routledge. 
Shamdasani, S. (2011). Jung e a Construção de uma Psicologia Moderna: O Sonho de uma Ciência. Aparecida - SP: Editora Ideias e Letras.

Shamdasani, S. (2012). Introdução. In. C. Jung, O livro vermelho: Liber Novus. Petrópolis: Vozes.

Vieira, A. G. (2006). A função da história e da cultura na obra de C. G. Jung. Aletheia, (23), 89-100. Recuperado em 14 de dezembro de 2020, de http://pepsic.bvsalud.org/scielo.php?script=sci_arttext\&pid=S1413$03942006000200010 \& \mathrm{lng}=$ pt\&tlng $=$ pt.

Waldron, S. (2003). Cultural property and the dilemma of the collective unconscious. Quadrant, XXXIII, 35-49.

\section{CONFLITOS DE INTERESSE}

Os autores declaram não haver conflito de interesse no trabalho apresentado

\section{SOBRE OS AUTORES}

Cauan Esplugues Silva é Psicólogo pela Pontifícia Universidade Católica do Paraná (2016) e Mestre em Psicologia pela Universidade Federal do Paraná (2019). Psicólogo clínico e Professor na pósgraduação em Psicologia da Unibrasil.

e-mail: cauan.es@outlook.com

(2) https://orcid.org/0000-0001-6264-7035

Carlos Augusto Serbena é Psicólogo, Professor Associado do Departamento de Psicologia da Universidade Federal do Paraná (UFPR) e integrante permanente do Programa de Pós-Graduação em Psicologia da UFPR na linha de Psicologia Clínica. Doutor em Ciências Humanas pela Universidade Federal de Santa Catarina (2006), mestre em Psicologia e Sociedade pela Universidade Federal de Santa Catarina (1999). Participa do GT da ANPPEP Epistemologia da Psicologia Analítica e Interfaces. Tem feito pesquisas, orientado trabalhos, ministrado disciplinas e realizado supervisão clínica em Psicologia Analítica, psicoterapia, fundamentos e interfaces com a fenomenologia.

e-mail: caserbena@gmail.com

(1) https://orcid.org/0000-0001-5568-839X

${ }^{1}$ Eranos foi fundado por Olga Froebe-Kapteyn, em 1933. Eram conferências realizadas anualmente por intelectuais de diferentes áreas, como a psicologia profunda, história, ciências naturais, física, química, religiões comparadas, entre outros. 